where $A_{0}$ is the group of all finite, even permutations, $\Sigma_{0}$ the group of all finite permutations and for each cardinal number $\boldsymbol{\aleph}$ the group $\Sigma_{\boldsymbol{N}}$ consists of all correspondences involving at most $\boldsymbol{N}$ elements. The preceding considerations are sufficient (as pointed out by the referee) to show that each of them consists only of its own commutators with the obvious exception of $\Sigma_{0}$ whose commutators form $A_{0 .}$ ]

YALE UNIVERSITY

\title{
NOTE ON A LEMMA OF A. W. GOODMAN
}

\author{
E.G.-RODEJA F.
}

In a recent and interesting paper on $p$-valent functions by $A . W$. Goodman we find the following lemma. ${ }^{1}$

LEMmA 1. For all integers $n \geqq k \geqq 1$

$$
\sum_{m=k}^{n} \frac{(-1)^{m+k} 2 n k}{(m+k) !(m-k) !} \prod_{\alpha=1}^{m-1}\left(n^{2}-\alpha^{2}\right)=\delta_{n}^{k},
$$

and for all integers $n>k \geqq 1, \beta \geqq 1$,

$$
\sum_{m=k}^{n}(-1)^{m} \prod_{\alpha=1}^{m-1}\left(n^{\beta}-\alpha^{\beta}\right) \prod_{\alpha=m+1}^{n}\left(\alpha^{\beta}-k^{\beta}\right)=0 .
$$

Here the empty product is unity by definition.

The identity (1), which is the first half of the lemma, has been used in the said paper, while (2) is stated only as a generalization of (1).

In this note we intend to establish the identities (13) and (14) (where $F_{n}$ and $G_{n}$ are arbitrary functions of the natural number $n$ ), including the afore mentioned as particular cases; and for their demonstration we shall follow a method in part similar to the one used in the paper quoted.

Let $A_{n}^{(m)}$ be a function of the natural numbers $n$ and $m$ satisfying the conditions (a) $A_{n}^{(m)}=0$ if $n<m$, (b) $A_{n}^{(m)}=1$ if $n=m$.

$H(p, k, n)$ is defined by

Received by the editors March 6, 1950.

1 A. W. Goodman, On some determinants related to p-valent functions, Trans. Amer. Math. Soc. vol. 63 (1948) pp. 175-192. 
(3)

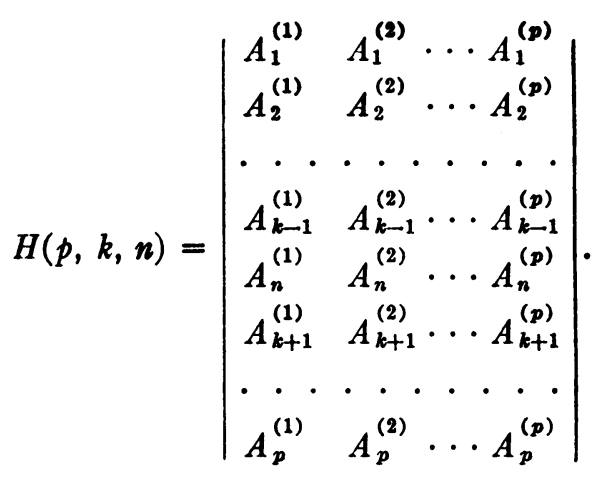

If $a_{i}, i=1,2, \cdots, p$, are indeterminate and

$$
b_{n}=\sum_{m=1}^{p} a_{m} A_{n}^{(m)}, \quad n=1,2, \cdots,
$$

the following identities are verified

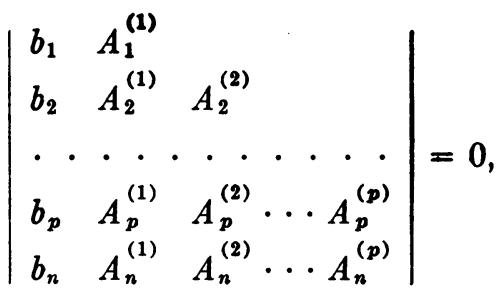

determinants that, developed by their first column, lead us to

$$
b_{n}=\sum_{k=1}^{p} b_{k} H(p, k, n) .^{2}
$$

From the first $m$ relations $(4), m \leqq p$, we get:

$$
a_{m}=b_{m}-\sum_{k=1}^{m-1} b_{k} H(m-1, k, m) \text {. }
$$

Using (7) in (4), we have

$$
\begin{aligned}
b_{n} & =\sum_{m=1}^{p} A_{n}^{(m)}\left[b_{m}-\sum_{k=1}^{m-1} b_{k} H(m-1, k, m)\right] \\
& =\sum_{k=1}^{p} b_{k}\left[A_{n}^{(k)}-\sum_{m=k+1}^{p} A_{n}^{(m)} H(m-1, k, m)\right],
\end{aligned}
$$

2 With the direct obtainment of relation (6), Lemma 1, apart from its intrinsic value, is unnecessary in Goodman's paper. 
and comparing with (6), we have

$$
H(p, k, n)=A_{n}^{(k)}-\sum_{m=k+1}^{p} A_{n}^{(m)} H(m-1, k, m) .
$$

For $n \leqq p$, if we keep in mind the value of $H(p, k, n)$,

$$
\delta_{n}^{k}=A_{n}^{(k)}-\sum_{m=k+1}^{n} A_{n}^{(m)} H(m-1, k, m),
$$

where the superior limit of the sum has been modified according to condition (a) for $A_{n}^{(m)}$.

If $F_{n}=F(n)$ and $G_{n}=G(n)$ are functions of the natural number $n$, $G_{m} \neq 0$ and $F_{p} \neq F_{q}$ if $p \neq q$, the function ${ }^{3}$

$$
A_{n}^{(m)}=\frac{G_{n} \prod_{\alpha=1}^{m-1}\left(F_{n}-F_{\alpha}\right)}{G_{m} \prod_{\alpha=1}^{m-1}\left(F_{m}-F_{\alpha}\right)}
$$

satisfies conditions (a) and (b). In this case $H(p, k, n)$ contains in the $k$ th row, besides the factor $G_{n}$ to be found in every element, a polynomial in $F_{n}$ of degree at most $p-1$. But $H(p, k, n)$ vanishes for $n=1,2, \cdots, k-1, k+1, \cdots, p$ and therefore $F_{1}, F_{2}, \cdots, F_{k-1}$, $F_{k+1}, \cdots, F_{p}$ are roots of this polynomial. For $n=k$, by conditions (a) and (b), $H(p, k, n)=1$.

By the preceding considerations,

$$
H(p, k, n)=\frac{G_{n} \prod_{\alpha=1}^{k-1}\left(F_{n}-F_{\alpha}\right) \prod_{\alpha=k+1}^{p}\left(F_{n}-F_{\alpha}\right)}{G_{k} \prod_{\alpha=1}^{k-1}\left(F_{k}-F_{\alpha}\right) \prod_{\alpha=k+1}^{p}\left(F_{k}-F_{\alpha}\right)} .
$$

If we use (11) and (12) in (10), it follows, for $n \geqq k \geqq 1$, that

$$
\delta_{n}^{k}=\sum_{m=k}^{n}(-1)^{m+k} \frac{G_{n} \prod_{\alpha=1}^{m-1}\left(F_{n}-F_{\alpha}\right)}{G_{k} \prod_{\alpha=1}^{k-1}\left(F_{k}-F_{\alpha}\right) \prod_{\alpha=k+1}^{m}\left(F_{\alpha}-F_{k}\right)},
$$

${ }^{3}$ There is no real gain in generality here when the factor $G_{n} / G_{m}$ is introduced, since an inspection of equation (13) shows that this factor is common to all terms of the sum and may be dropped when $n \neq k$. When $n=k$ the factor is one. We have included this term merely to maintain a strict similarity between the proof of Lemma 1 and our generalization. 
an identity which, for $n>k \geqq 1$, can be transformed into

$$
\sum_{m=k}^{n}(-1)^{m} \prod_{\alpha=1}^{m-1}\left(F_{n}-F_{\alpha}\right) \prod_{\alpha=m+1}^{n}\left(F_{\alpha}-F_{k}\right)=0 .
$$

The identity (13), for $G_{n}=n, F_{n}=n$, becomes the well known one between the binomial coefficients

$$
\sum_{m=k}^{n}(-1)^{m+k} C_{n, m} C_{m, k}=\delta_{n}^{k} .
$$

For $G_{n}=n, F_{n}=n^{2}$, we find (1).

The identity (14), from which we get (2) for $F_{n}=n^{\beta}$, can also be deduced, for $s=n$, from the identity

$$
\begin{aligned}
\sum_{m=k}^{s}(-1)^{m} \prod_{\alpha=k}^{m-1}\left(F_{n}-F_{\alpha}\right) & \prod_{\alpha=m+1}^{n}\left(F_{\alpha}-F_{k}\right) \\
& =(-1)^{8} \prod_{\alpha=k+1}^{8}\left(F_{n}-F_{\alpha}\right) \prod_{\alpha=s+1}^{n}\left(F_{\alpha}-F_{k}\right),
\end{aligned}
$$

which is easily demonstrable by induction with respect to $s$.

SANTiago de Compostela 